\title{
Un settembre molto idrogeologico
}

\section{A "very hydrogeologic" September}

Daniela Ducci - Presidente IAH Italia - presidente@iahitaly.it

Paolo Fabbri - Membro commissione internazionale "Mineral and Thermal Waters"

Marco Petitta - Vice Presidente IAH internazionale - Europa (centrale e occidentale)

Sergio Rusi - Segretario IAH Italia

Keywords: IAH Events, congresses, conferences.

Parole chiave: eventi IAH, congressi, conferenze.

Nel settembre 2019 si sono avute molte occasioni di incontri e di riflessioni scientifiche per gli idrogeologi italiani.

La rassegna degli eventi IAH o patrocinati da IAH che si sono svolti in questo periodo comincia con il congresso congiunto SIMP-SGI-SOGEI (Società Italiana di Mineralogia e Petrologia, Società Geologica Italiana e Società Geochimica Italiana) dal titolo "Il tempo del pianeta Terra e il tempo dell'uomo: Le geoscienze fra passato e futuro" che si è tenuto a Parma dal 16 al 19 settembre 2019 (Fig. 1). La sessione di idrogeologia S26 patrocinata da IAH Italy "Approaches for evaluation and protection of groundwater resources", avente come conveners Daniela Ducci, Emma Petrella e Stefania Stevenazzi, ha ricevuto 17 contributi, di cui 4 presentati oralmente. Il primo è stata la keynote presentata dal dott. Di Curzio su "Stationary and non-stationary geostatistics to model 3-D bydraulic conductivity distribution", in cui l'autore ha illustrato tra l'altro metodologie innovative per la valutazione della conducibilità idraulica da dati di prove penetrometriche statiche. Gli altri lavori presentati hanno riguardato prevalentemente studi isotopici, analisi statistiche in idrogeologia e geotermia.

Dal 18 al 20 settembre si è tenuto a Ferrara il RemTech Expo, l'unico evento internazionale permanente specializzato sulle bonifiche, rischi ambientali e naturali, sicurezza, manutenzione, riqualificazione, rigenerazione del territorio, cambiamenti climatici e chimica circolare. La kermesse ha visto la partecipazione ufficiale del Consiglio Nazionale dei Geologi, che ha organizzato gli Stati Generali della Geologia. I temi di

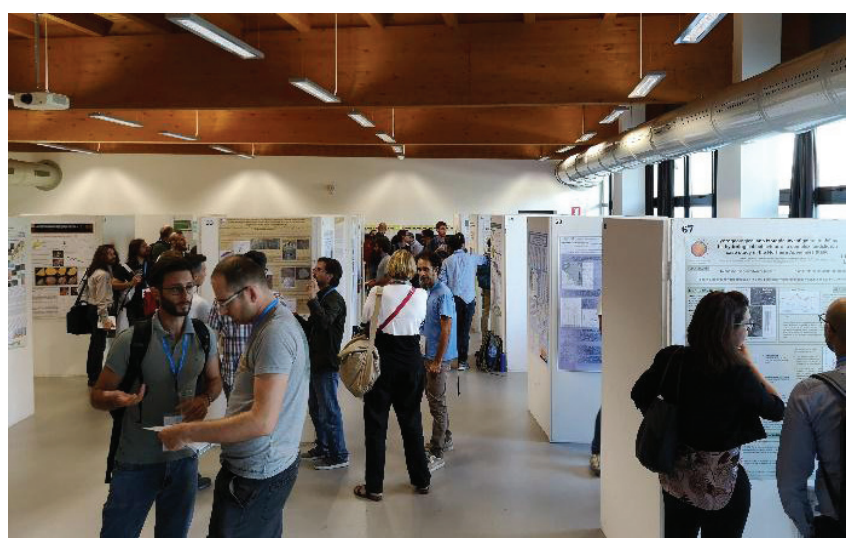

Fig.1 - La sessione poster del convegno SIMP-SGI-SOGEI (foto di Sergio Rusi). Fig. 1 - The poster session of the SIMP-SGI-SOGEI Congress (taken by Sergio Rusi). discussione sono stati molteplici, affrontati in modalità moderna, attraverso la creazione di sei distinti tavoli di lavoro, ad ognuno dei quali una decina di specialisti ha portato il proprio contributo specifico. Al tavolo "Rischi e Risorse" buona parte dei lavori sono stati dedicati al tema dell'acqua, attraverso confronto di conoscenze, esperienze e criticità. Ne sono emersi spunti interessanti, tra i quali vanno segnalati la riconosciuta necessità di mettere a fattor comune le conoscenze spesso disperse o non disponibili, e l'intenzione di organizzare una Conferenza Nazionale sull'Acqua in cui far convergere $\mathrm{i}$ vari soggetti istituzionalmente investiti della gestione della risorsa. Per ambedue le iniziative si è riconosciuto il ruolo di catalizzatore che il Consiglio Nazionale dei Geologi può e deve rivestire. Nel pomeriggio le idee scaturite dai diversi tavoli sono state omogeneizzate e riassunte ad una più ampia platea di partecipanti. Anche alla luce di queste rilevanti novità, per la IAH la collaborazione con il CNG continua ad essere di fondamentale importanza.

Dal 19 al 20 settembre, si è tenuto a Matera l'XI Congresso AIGA Giovani Ricercatori con due sessioni di idrogeologia le cui interessanti keynotes sono state tenute da ricercatori iscritti alla nostra associazione. Maurizio Polemio, CNR-IRPI di Bari, ha tenuto una relazione dal titolo "Monitoring and management of coastal groundwater resources" e Fulvio Celico, Università di Parma, ha presentato "Approccio metagenetico-isotopico per il monitoraggio di fluidi in giacimenti petroliferi".

Alla fine del convegno sono state premiate le migliori presentazioni ed i migliori posters. Un nostro iscritto Filippo Torresan, dottorando all'Università di Padova, ha ricevuto il premio per la miglior presentazione della sessione di idrogeologia "Chimismo e potenzialità delle acque sotterranee" (Fig. 2).

Si è appena concluso Il $46^{\circ}$ Congresso internazionale IAH del 2019, che si è tenuto a Malaga, in Spagna, dal 22 al 27 settembre. Il Congresso, del cui Comitato Scientifico facevano parte D. Ducci e V. Re, si articolava in dieci topics sul tema: "Groundwater Management and Governance: Coping with Water Scarcity", e nel dettaglio:

- $\quad$ T1 Groundwater assessment and management;

- $\quad \mathrm{T} 2$ Groundwater and climate change;

- T3 Groundwater sustainability and governance;

- T4 Groundwater footprint and virtual water; 


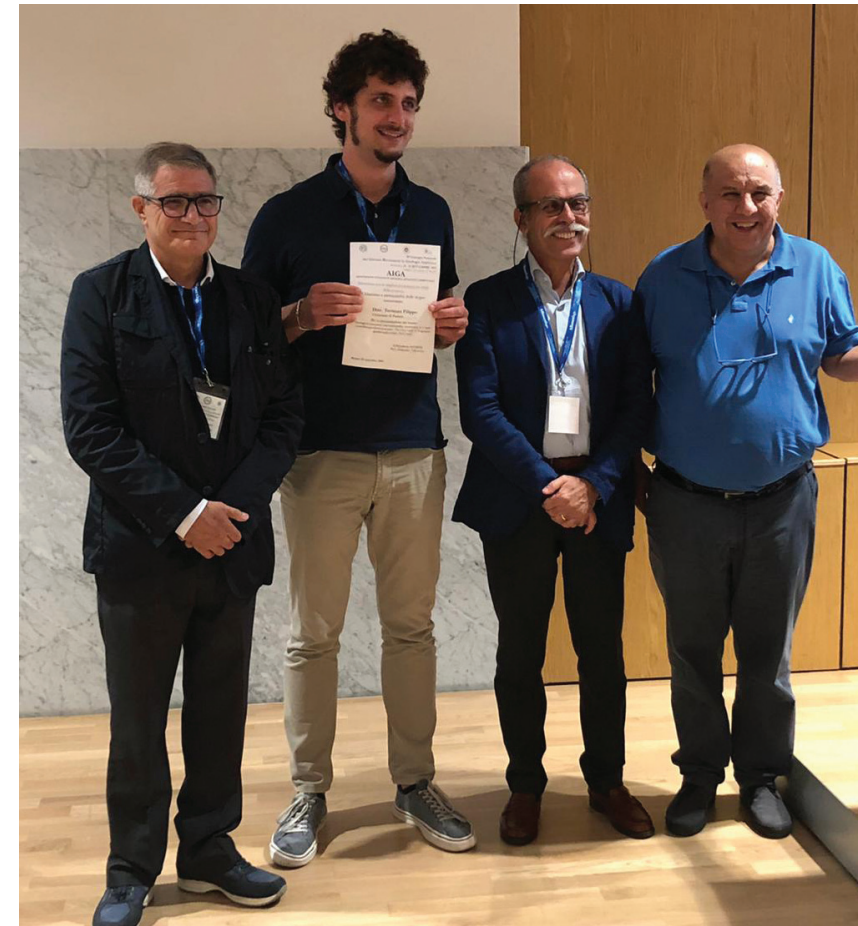

Fig.2 - Il socio IAH, Filippo Torresan, premiato al convegno AIGA di Matera, con il Presidente AIGA, Prof. Calcaterra, e gli organizzatori, Proff. Sdao e Simeone (foto di Paolo Fabbri).

Fig. 2 - The IAH member, Filippo Torresan, awarded at the AIGA Congress in Matera, with the Aiga President, Prof. Calcaterra, and the professors Sdao and Simeone (taken by Paolo Fabbri).

- $\quad$ T5 Tools, methods and models to study groundwater;

- $\quad$ T6 Groundwater, wetlands and natural heritage;

- T7 Karst Hydrogeology;

- T8 Groundwater quality and pollution processes;

- T9 Groundwater and socio-economic development in Latin-America;

- T10 Urban Hydrogeology
I partecipanti sono stati numerosi (770), provenienti da 83 diverse nazioni: in primis la Spagna con poco meno di 120 partecipanti, seguita dalla Cina con più di 50 partecipanti, quindi dalla Francia e dalla Germania. L'Italia è stata quinta come partecipazione con 33 rappresentanti (Fig. 3), che hanno presentato 38 abstracts, di cui 23 selezionati come presentazioni orali.

Il congresso IAH ha presentato 12 keynote lectures, tutte con un ottimo successo di pubblico, di rilievo quelle di Mattew Rodell della NASA, che ha fatto una dettagliata disamina di tutte le possibilità attuali di osservazione da satellite dei cambiamenti climatici, di John Cherry, che ha parlato dell'opportunità di diffondere la cultura idrogeologica tramite ebooks gratuiti. Lultima keynote, quella dedicata a un membro ECHN è stata del nostro socio Stefano Viaroli (Fig. 4), che ha intrattenuto il pubblico presente con uno stimolante argomento: "The Good, the Bad and the Bottled: considerations of the effects of our habits on the water and environmental protection". Al termine della brillante Keynote, il socio Viaroli ha invitato i presenti a partecipare al prossimo evento internazionale MinWat 2020 (vedi sotto) coorganizzato da IAH Italy.

Lorganizzazione delle sessioni orali nelle varie sale del Trade Fairs and Congress Center (FYCMA) è stata molto buona, così come quella dei lunches e dei rinfreschi-poster, che hanno consentito intensi momenti di socializzazione tra i partecipanti.

Nella giornata di martedì l'usuale assemblea IAH ha aggiornato i soci sulle ultime novità, e tra queste una davvero entusiasmante per IAHItaly: la creazione di un nuovo Network, "Socio-Hydrogeology Network", diretto da Viviana Re, che da anni sviluppa questa tematica nell'ambito dei suoi studi.

Il terzo giorno del Congresso è stato dedicato ai field trips, che avevano come oggetto l'illustrazione di casi di studio stimolanti dal punto di vista scientifico e naturalistico (Karst, wetlands e aree costiere) su realtà idrogeologiche spagnole di grande interesse, alcune delle quali di tipo inconsueto essendo relative a contesti climatici semiaridi e non mediterranei come ci si potrebbe aspettare. Di particolare interesse il field

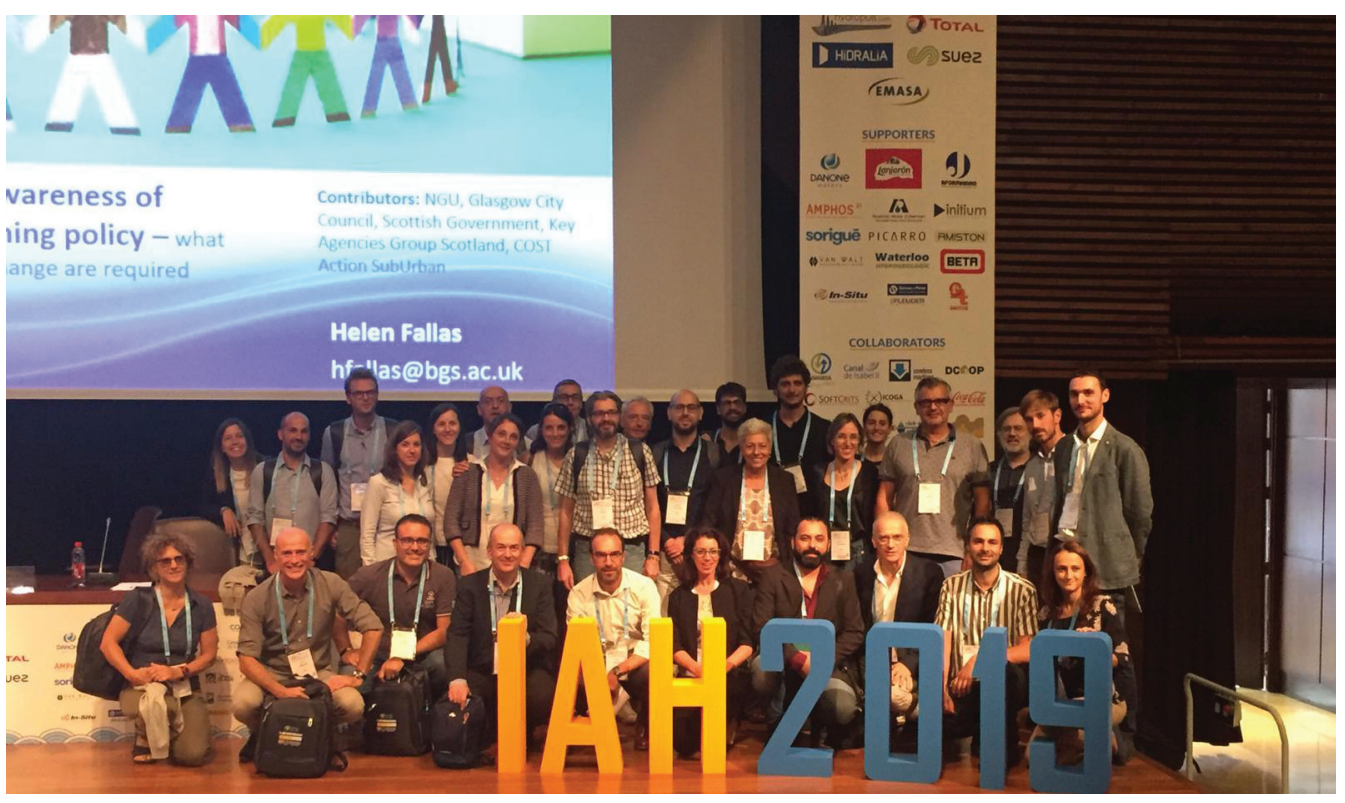

Fig.3 - La delegazione italiana al $46^{\circ}$ Congresso IAH (foto di Francesco La Vigna).

Fig. 3 - The Italian delegation at the $46^{\text {th }}$ IAH Congress (taken by Francesco La Vigna). 


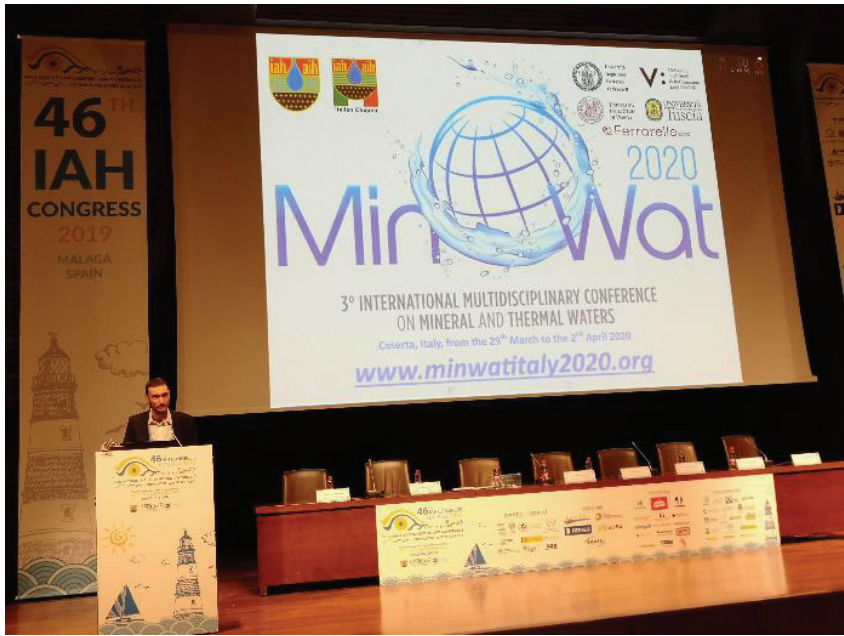

Fig. 4 - Il socio Stefano Viaroli durante la keynote lecture al $46^{\circ}$ Congresso IAH (foto di Sergio Rusi).

Fig. 4 - The member Stefano Viaroli during the Keynote lecture at thr 46th IAH Congress (taken by Sergio Rusi).

trip 5 nella zona del Torcal de Antequera, un altipiano carsico di particolare bellezza e peculiari caratteristiche idrogeologiche.

Bisogna segnalare che, come sempre il Congresso è stato preceduto, nella giornata di sabato, dai Pre-congress Short Courses e nell'ambito di quello sul karst bydrogeology "HYDROKARST", Marco Petitta ha tenuto una Lecture.

Come sempre vi indichiamo di seguito i prossimi appuntamenti per la comunità idrogeologica che vedranno la presenza attiva del Comitato Italiano IAH.

A Bologna, nella sede della Regione Emilia-Romagna di Viale della Fiera, il prossimo 30 settembre si farà il punto sulla lotta al cambiamento climatici, durante il convegno "Cambiamenti climatici e acque sotterranee”. L'evento offrirà un momento di approfondimento e dibattito con il mondo della ricerca, la pubblica Amministrazione e i rappresentanti del mondo produttivo su un tema strategico quale le relazioni tra il cambiamento del clima e le possibili ripercussioni sulla disponibilità di risorsa idrica, con una particolare attenzione alle possibili tecniche di ricarica delle falde.

A Forte dei Marmi si terrà dal 2 al 5 ottobre il convegno "Gestione delle risorse idriche” promosso, oltre che dal Comune toscano, dall'Università di Pisa, dalla Scuola Sant'Anna e all'Igg/Cnr di Pisa. Particolare rilievo nell'organizzazione hanno avuto i soci Re e Rossetto, che saranno anche tra gli speaker con altri i soci di IAHItaly: Doveri, Ducci, Menichini, Petitta, Sapiano.

Guardando un po' più avanti, l'IAH Italia è fortemente coinvolta nell'organizzazione di MinWat2020 (www.minwatitaly2020.org), la terza Multidisciplinary Conference on Mineral and Thermal Waters, della IAH commission on Mineral and Thermal Waters (MTWC), che si terrà dal 29 marzo al 2 aprile 2020 a Caserta, nella splendida cornice di Palazzo Paternò.
Il comitato scientifico è composto da 23 esperti internazionali delle sessioni proposte che sono:

- Topic 1 - Hydrogeology and geochemistry of mineral and thermal waters - Keynote Lecture: Orlando Vaselli - University of Firenze;

- Topic 2 - Protection and management of mineral and thermal waters - Keynote Lecture: Patrick Lachassagne - DANONE;

- Topic 3 - Bottled mineral waters - Keynote Lecture: Patricia Fosserland - European Federation of Bottled Waters;

- Topic 4 - Health issues, balneology and balneotherapy Keynote Lecture: Umberto Solimene - President of the World Federation for Hydrotherapy and Climatotherapy (FEMTEC);

- Special Session: Italian Mineral and Thermal Waters, from research to sustainable management.

Quest'ultima sessione si svilupperà in una tavola rotonda che metterà a confronto il mondo della ricerca, dell'imprenditoria e di chi ha responsabilità normative nella gestione delle risorse idriche sotterranee.

Ci saranno inoltre due field trips, il primo al Parco Terme di Telese, una località a $30 \mathrm{~km}$ da Caserta le cui sorgenti sono apparsa dopo il terremoto del 1349 a fianco della più famosa sorgente di Grassano. Il secondo agli impianti di imbottigliamento delle acque bicarbonato calciche ricche in $\mathrm{CO}_{2}$ della Ferrarelle (main sponsor e co-organizzatore dell'evento), posti nei pressi di Riardo (Campania) $50 \mathrm{~km}$ a NW di Telese e a SE del vulcano di Roccamonfina.

Il Comitato organizzatore è composto dai soci Corniello (UniNA), Fabbri (UniPD), Paolucci (Ferrarelle spa) e Tedesco (UniCAMPANIA), mentre la Segreteria organizzativa da i soci Cuoco (UniCAMPANIA), Forte (UniNA) e Viaroli (UniROMA3).

Per aggiornamenti e maggiori dettagli su tutti gli eventi previsti vi invitiamo come sempre a visitare il sito www.iahitaly.it, e i social (Facebook e Twitter), dove troverete anche le locandine. . 